\title{
Implementation of Tissue Harmonic Synthetic Aperture Imaging on a Commercial Ultrasound System
}

Rasmussen, Joachim; Hemmsen, Martin Christian; Madsen, Signe Sloth; Hansen, Peter Møller; Nielsen, Michael Bachmann; Jensen, Jørgen Arendt

\section{Published in:}

Proceedings of IEEE International Ultrasonics Symposium

Publication date:

2012

Document Version

Early version, also known as pre-print

Link back to DTU Orbit

Citation (APA):

Rasmussen, J., Hemmsen, M. C., Madsen, S. S., Hansen, P. M., Nielsen, M. B., \& Jensen, J. A. (2012). Implementation of Tissue Harmonic Synthetic Aperture Imaging on a Commercial Ultrasound System. In Proceedings of IEEE International Ultrasonics Symposium IEEE.

\section{General rights}

Copyright and moral rights for the publications made accessible in the public portal are retained by the authors and/or other copyright owners and it is a condition of accessing publications that users recognise and abide by the legal requirements associated with these rights.

- Users may download and print one copy of any publication from the public portal for the purpose of private study or research.

- You may not further distribute the material or use it for any profit-making activity or commercial gain

- You may freely distribute the URL identifying the publication in the public portal 
Paper presented at the IEEE International Ultrasonics Symposium, Dresden, Germany, 2012:

\section{Implementation of Tissue Harmonic Synthetic Aperture Imaging on a Commercial Ultrasound System}

Joachim Hee Rasmussen ${ }^{1}$, Martin Christian Hemmsen ${ }^{1}$, Signe Sloth Madsen ${ }^{2}$, Peter Møller Hansen ${ }^{2}$, Michael Bachmann Nielsen², and Jorgen Arendt Jensen ${ }^{1}$

${ }^{1}$ Center for Fast Ultrasound Imaging,

Biomedical Engineering group, Department of Electrical Engineering, Bldg. 349, Technical University of Denmark, DK-2800 Kgs. Lyngby, Denmark

${ }^{2}$ Department of Radiology,

Copenhagen University Hospital, Rigshospitalet, DK-2100 Copenhagen, Denmark

To be published in Proceedings of IEEE International Ultrasonics Symposium, Dresden, Germany, 2012. 


\title{
Implementation of Tissue Harmonic Synthetic Aperture Imaging on a Commercial Ultrasound System
}

\author{
Joachim Hee Rasmussen $^{a}$, Martin Christian Hemmsen ${ }^{a}$, Signe Sloth Madsen ${ }^{b}$, Peter Møller Hansen ${ }^{b}$, \\ Michael Bachmann Nielsen ${ }^{b}$, and Jørgen Arendt Jensen ${ }^{a}$ \\ ${ }^{a}$ Center for Fast Ultrasound Imaging, Dept. of Elec. Eng., Bldg. 349, \\ Technical University of Denmark, DK-2800 Kgs. Lyngby, Denmark \\ ${ }^{b}$ Department of Radiology, Rigshospitalet, DK-2100 Copenhagen, Denmark
}

\begin{abstract}
This paper presents an imaging technique for synthetic aperture (SAI) tissue harmonic imaging (THI) on a commercial ultrasound system. Synthetic aperture sequential beamforming (SASB) is combined with a pulse inversion (PI) technique on a commercial BK 2202 UltraView system. An interleaved scan sequence that performs dynamic receive focused (DRF) imaging and SASB, both using PI, is implemented. From each acquisition four images can be created: DRF image, SASB image, tissue harmonic DRF image (DRF-THI), and tissue harmonic SASB image (SASB-THI). For SASB imaging, a fixed transmit and receive focus at $80 \mathrm{~mm}$ and an $F \#$ of 3 is applied. For DRF imaging, default scanner settings are used, which are a focus at $85 \mathrm{~mm}$ and $\mathrm{F} \#$ of 5.7 in transmit and a dynamic receive aperture with an $\mathrm{F} \#$ of 0.8 . In all cases a $2.14 \mathrm{MHz}$ one-and-ahalf cycle excitation transmit waveform is used. A BK 8820e 192 element convex array transducer is used to conduct scans of wire phantoms. The $-6 \mathrm{~dB}$ and $-20 \mathrm{~dB}$ lateral resolution is measured for each wire in the phantom. Results show that the $-6 \mathrm{~dB}$ lateral resolution for SASB-THI is as good as for DRF-THI except at the point of the virtual source. SASB-THI even shows $7 \%$ reduction in -6 dB lateral resolution for the deepest wire at $100 \mathrm{~mm}$. The $-\mathbf{2 0}$ $\mathrm{dB}$ resolution for $\mathrm{SASB}-\mathrm{THI}$ at $[25,50,75,100] \mathrm{mm}$ was reduced by $[5,0-34,11] \%$ compared to DRF-THI, which shows, that except for the point of the virtual source, the lateral resolution was improved by SASB-THI. A successful implementation of SASB-THI was achieved on a commercial system, which can be used for future pre-clinical trials.
\end{abstract}

\section{INTRODUCTION}

In ultrasound B-mode imaging, several techniques to improve spatial resolution have been developed over the past decades. Tissue harmonic imaging has long been used to image difficult regions with an increased contrast and resolution. One of the most common THI techniques is the pulse inversion (PI) scheme, which is implemented in many ultrasound systems today. The PI technique can be combined with a variety of focusing techniques. One focusing technique, that obtains high and depth independent spatial resolution is synthetic aperture imaging (SAI). This paper presents an imaging technique for synthetic aperture tissue harmonic imaging (SAI-THI) on a commercial ultrasound system. A parameter study is presented which shows the optimal positions for focal depth and F\# for SAI-THI. Furthermore, a comparison of the spatial resolution between SAI-THI and a common THI technique is presented. Finally, a simultaneously acquired in-vivo image using the two techniques is presented and compared.

\section{THEORY}

\section{A. Pulse Inversion}

In THI the purpose is to image a single harmonic component of a pulse response, instead of the fundamental component. A PI technique can be used to isolate the 2nd harmonic component from its neighboring harmonic components and enhance it [1], [2]. In this technique two pulses (regular pulse, $180^{\circ}$ phase shifted pulse) are transmitted in turn. While a $180^{\circ}$ phase shift can be detected for the fundamental component of the received signal, a corresponding $360^{\circ}$ and $540^{\circ}$ phase shift can be seen for the 2nd and 3rd harmonic components respectively [3], [4]. To suppress the odd order harmonics, each correlated pair (regular and phase shifted) of the received responses are summed. Harmonic components that are in phase (all even harmonics) will double in amplitude, while out of phase components (all odd harmonics) will cancel out (see Fig. 1). This separates and enhances the 2nd harmonic component, which in turn then can be filtered and used for imaging.

\section{B. Synthetic Aperture Sequential Beamforming}

Traditionally, SAI utilizes only one active transducer element in transmit. Because the transmitted acoustic energy in this case is very low, SAI is not capable of producing harmonic components for THI. Synthetic aperture sequential beamforming (SASB) is a SAI technique, which uses virtual sources and a dual stage beamforming approach to significantly reduce the computational load compared to SAI [5], [6]. A fixed transit and receive focal point is used in the first stage beamformer to create a set of focused scan lines. Each image point is potentially represented in several scan lines and can be used to improve resolution (see Fig. 2). The second stage beamformer combines the contributing first stage scan lines into an image, which has higher and more depth independent 


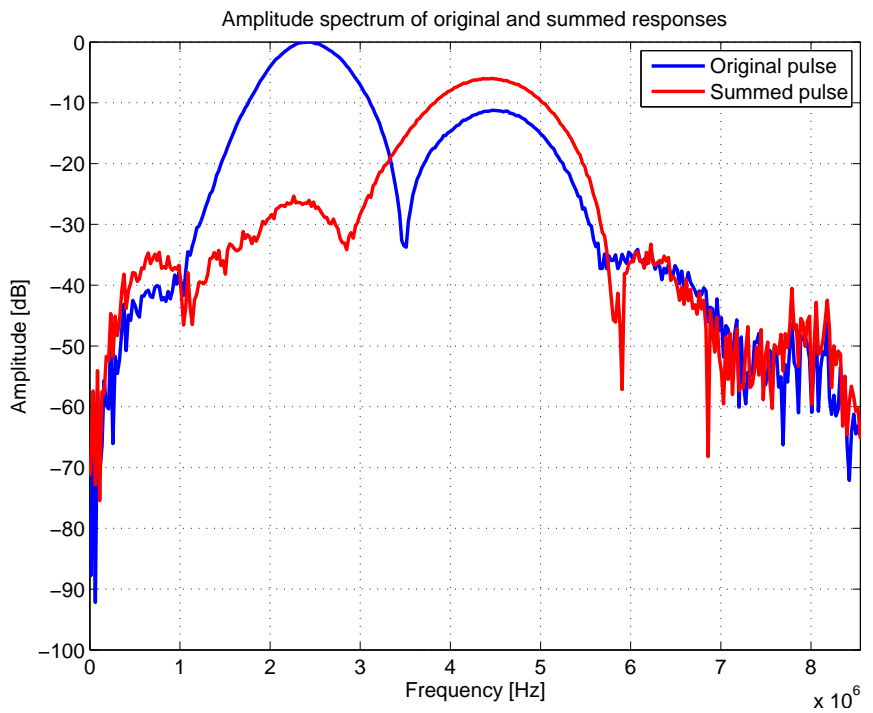

Figure 1. Spectrum of received RF signals. The blue curve shows the spectrum of a received response, while the red curve shows the spectrum after pulse summation with the inversed response. The 2 nd harmonic component at $4.24 \mathrm{MHz}$ is enhanced by approximately $6 \mathrm{~dB}$, while the fundamental component at $2.14 \mathrm{MHz}$ is attenuated by approximately $25 \mathrm{~dB}$.

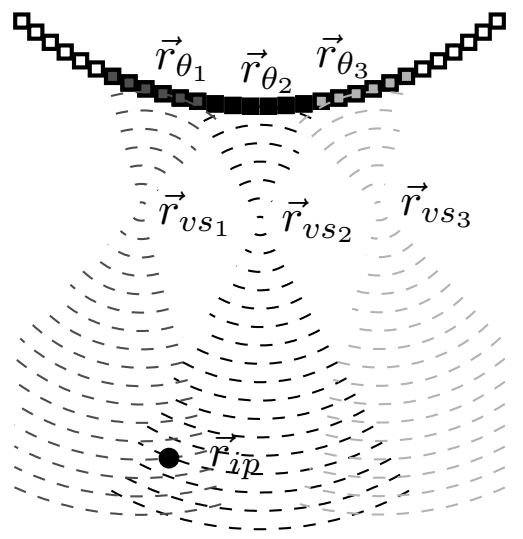

Figure 2. Three emissions for first stage SASB. A scan line is created with reference $r \theta$ and a virtual source at $r_{v s}$ in each emission. The dotted lines show the image information at spatial positions that are found in each focused scan line. A single image point, $r_{i p}$ may be represented in several focused scan lines as shown by the black dot. The second stage beamformer determines which scan lines contain information from the same point and sums the contributions into one image. Figure taken from [6]

lateral resolution (see Fig. 3). The advantage of SASB, besides the improvements in lateral resolution, is that the transmitted acoustic energy is sufficient to produce harmonic components for THI [7]. A combination of SASB and THI can therefore be implemented on a commercial ultrasound system.

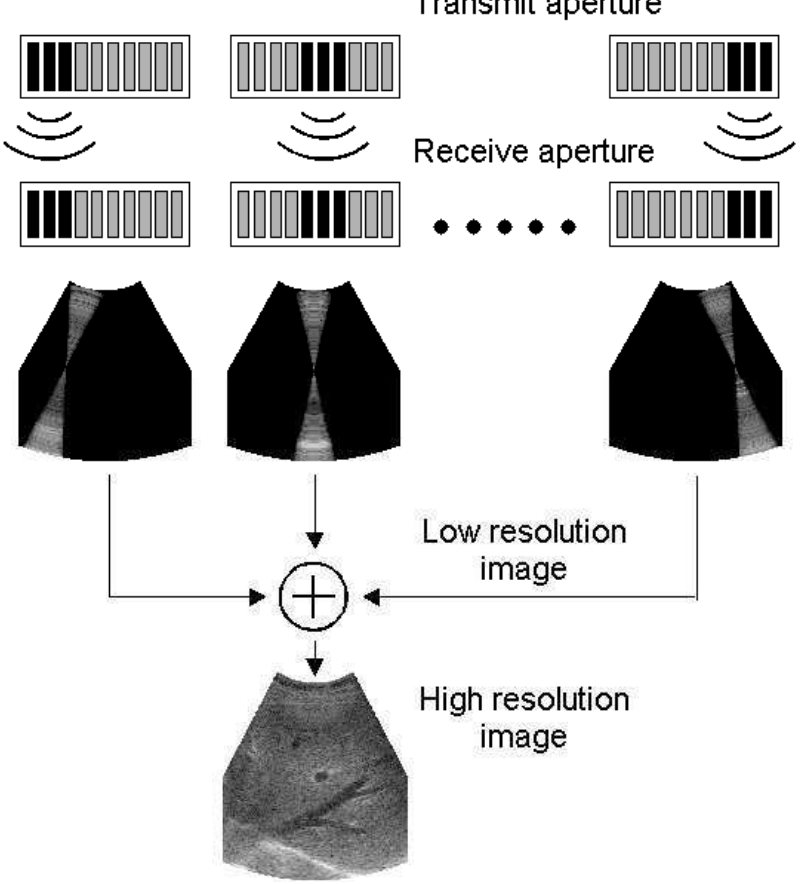

Figure 3. Schematic illustration of the dual stage beamformer. A number of acquisitions using fixed focus in transmit and receive are obtained. The received responses are beamformed along the scan line direction using the delay profile used in transmit (first stage beamforming). The resulting image data is then beamformed again (second stage beamforming) using a focus at each pixel in the image. This produces a set of low resolution images which are then summed coherently to form a single high resolution image. Figure taken from [6]

\section{TISSUE HARMONIC SYNTHETIC APERTURE IMAGING ON COMMERCIAL ULTRASOUND SYSTEM}

A tissue harmonic SASB (SASB-THI) scan sequence is implemented on a commercial ultrasound system. A BK 2202 UltraView ultrasound system (UltraView) is equipped with a research interface that allows the user to adjust and control many scan parameters such as focal depth, F\#, excitation waveform, etc. The research interface also allows the user to access and extract image IQ-data. The system has a collection of pre-defined settings for different imaging techniques. The default imaging technique, when conducting abdominal scans, is dynamic receive focusing tissue harmonic imaging (DRFTHI). This technique is used as a golden standard to compare SASB-THI against. In this case, the research interface is used to conduct a PI scan sequence using both DRF and SASB imaging simultaneously and extract DRF beamformed and SASB first stage image data. A 192 element BK 8820e convex array transducer is used. The default settings for DRF and DRF-THI can be found in Table I. A fixed focus is used for SASB for both transmit and receive, while an expanding aperture is used for DRF imaging. Transmit apodization for SASB and DRF is a Boxcar, while receive apodization is a Gauss for both. A total of 192 image lines each with two emissions (one for each PI pulse) is made for both SASB and DRF imaging. The scan sequence is such that $2 \times 192$ emissions 
are made first for every scan line for SASB with the PI emissions interleaved. Next, a second set of 2x192 emissions are made for all DRF scan lines. This ensures minimum tissue movement between the PI emissions. A one-and-a-half cycle excitation pulse with center frequency of $2.14 \mathrm{MHz}$ is used for both techniques. The collection of data acquired in one frame can be used for two linear B-mode images (one using SASB; one using DRF) and two 2nd harmonic B-mode images (SASB-THI and DRF-THI)

\section{A. Parameter Study}

A series of experiments is conducted in order to determine the optimal position of the focal point and value of the F\# for SASB-THI. Measurements of the lateral resolution and point spread functions are conducted using SASB-THI on a water filled phantom with 6 equidistant wires. The focal points are varied from $50-120 \mathrm{~mm}$ in steps of $10 \mathrm{~mm}$ and the $\mathrm{F} \#$ from 1.0-5.0 in steps of 0.5. For each combination of settings the $-6 \mathrm{~dB}$ lateral resolution (FWHM) is measured and plotted as function of depth for each applied F\#. Fig. 4 shows the measured FWHM values for each wire for every applied F\# at $80 \mathrm{~mm}$ focal depth. The plot can be used to determine which focal depth and which F\# can produce the best lateral resolution. The best lateral resolution is found where the FWHM values are low (good lateral resolution) and do not vary much with depth (depth independent lateral resolution). As seen from Fig. 4, the FWHM generally increases more with image depth as the F\# increases. An F\# of 2 produces the lowest and most constant scores of FWHM in this plot and could potentially be the best F\# at this focal depth. However, the level of side lobes must also be taken into account when choosing a low F\#.

Fig. 5 shows the point spread functions at $65 \mathrm{~mm}$ depth in the wire phantom for different F\# 's. It is seen from the plots that the value of F\# has great influence on the shape of the PSF. A low F\# results in narrow main lobe with high levels of side lobes, while a high F\# results in more wide main lobe and no side lobes. This is because the F\# determines the width of the apodization curve applied in transmit and receive. In transmit, the apodization curve is always a Boxcar, while in receive, a Gauss curve is used. When a low F\# is applied, the Gauss apodization curve that is used in receive, exceeds the actual width of the active aperture causing the edges of the Gauss curve to be cut off. In case of a very low F\#, a greater part of the Gauss curve is cut off, causing the apodization curve to take the shape of a square or Boxcar, resulting in higher levels of side lobes. At higher values of the F\# , the Gauss apodization curve fits the active aperture and side lobes are reduced. The difference is clearly seen in the top and bottom plot of Fig. 5, where F\#'s are 1.0 and 5.0 respectively. In the top plot, the PSF has a very narrow main lobe, but also has distinct side lobes, while the PSF in the bottom has a wider main lobe, and no side lobes. The PSF for F\# of 3.0 is shown in the middle plot of Fig. 5. Here, a compromise between main

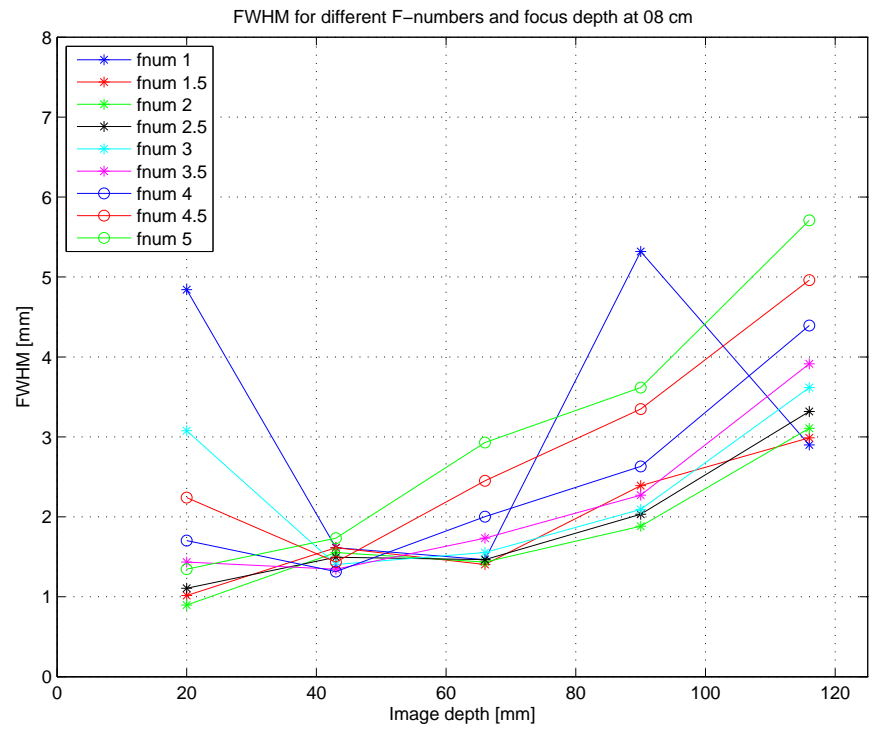

Figure 4. The $-6 \mathrm{~dB}$ lateral resolution (FWHM) measured for wires in wire phantom using different values of $\mathrm{F \# .} \mathrm{The} \mathrm{transmit} \mathrm{and} \mathrm{receive} \mathrm{focal} \mathrm{point}$ is set to $80 \mathrm{~mm}$ in all cases. The best value for $\mathrm{F} \#$ is the one that results in low and almost uniform FWHM. This must however, be held against the level of side lobes in each case, because lower F\#'s result in higher levels of side lobes.

lobe width and levels of side lobes has been made to achieve optimal spatial resolution without artifacts from side lobes.

\section{B. Performance study}

This study is conducted in order to determine the performance of SASB-THI compared to DRF-THI on the UltraView system. This is done by measurements and comparisons of the lateral resolution and by visual inspection of B-mode images of wire phantoms and in-vivo B-mode images. The settings used for DRF-THI and SASB-THI are shown in Table I. The settings chosen for DRF-THI are the default settings applied by the UltraView system, while the settings for SASB-THI where determined from the results in Sec. III-A.

One frame of the wire phantom and one in-vivo frame is acquired using the scan sequence on the system. DRF and first stage SASB image data are separated and extracted from the frame, inverted image lines are summed (pulse summation), and SASB data are beamformed on an external PC. For the wire phantom scan, the FWHM and $-20 \mathrm{~dB}$ lateral resolution (FWOTM) for each visible wire is measured. For the in-vivo scan, a visual comparison between the two images is made.

1) Comparison of Lateral Resolution: Fig. 6 shows the measured lateral resolutions for all four image techniques on the wire phantom. It is seen from the figures, that THI generally produces better lateral resolution for both SASB and DRF. It is also seen that, SASB-THI produces almost the same lateral resolution as DRF-THI except near the focal point, where the lateral resolution of SASB-THI is worse than DRFTHI. However, SASB-THI shows a 7\% reduction in FWHM 

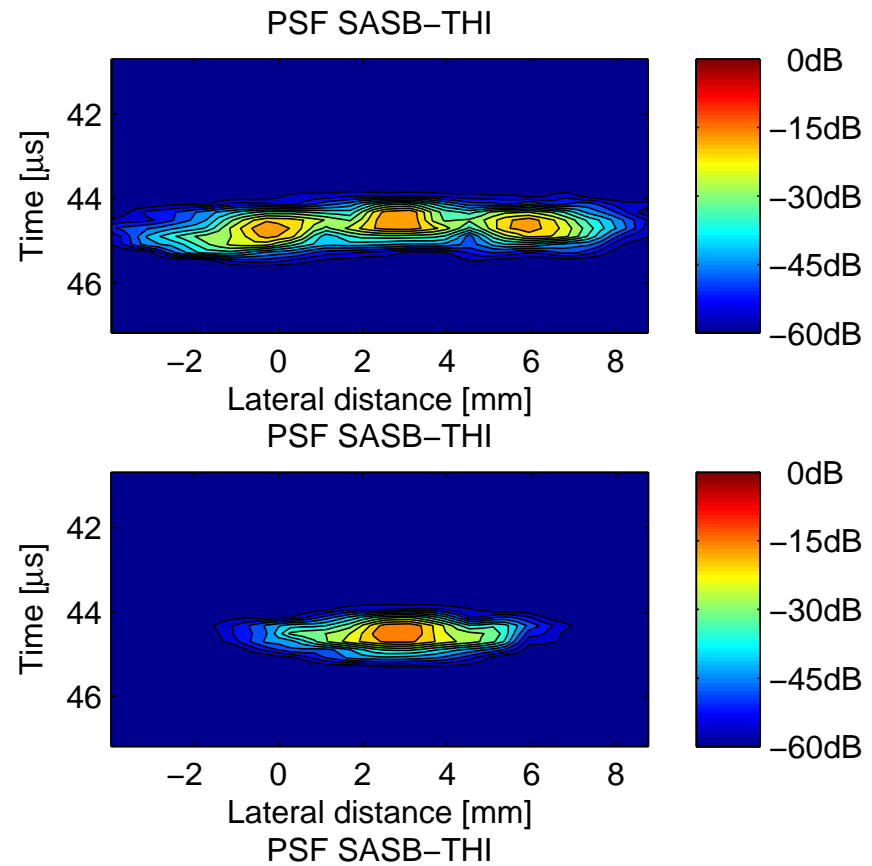

$0 \mathrm{~dB}$

$-15 d B$

$-30 \mathrm{~dB}$

$-45 \mathrm{~dB}$

$-60 d B$
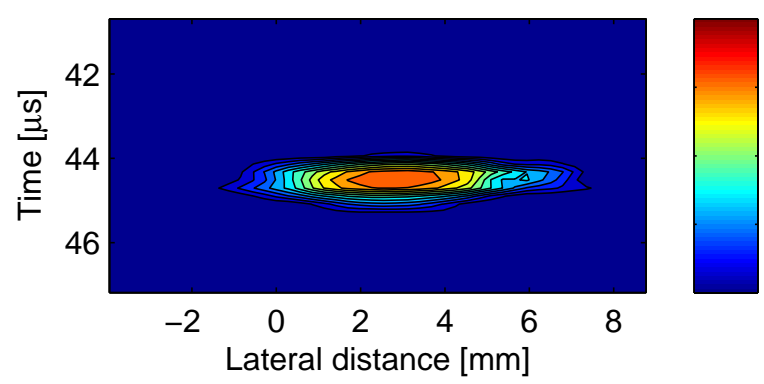

$0 \mathrm{~dB}$

$-15 \mathrm{~dB}$

$-30 \mathrm{~dB}$

$-45 d B$

$-60 \mathrm{~dB}$

Figure 5. Point spread function (PSF) for wire at $65 \mathrm{~mm}$ depth using SASBTHI illustrated in $6 \mathrm{~dB}$ contour plot. Focus is set to $80 \mathrm{~mm}$ and F\# is 1.0 , 3.0 , and 5.0 from top to bottom respectively.

Table I

PARAMETERS USED FOR SASB AND DRF ON THE ULTRAVIEW RESEARCH INTERFACE SCANNER.

\begin{tabular}{lll}
\hline Scanner Parameters & Value & \\
\hline Speed of sound & $1492 \mathrm{~m} / \mathrm{s}$ & \\
Transmit center frequency & $2.14 \mathrm{MHz}$ & \\
Sampling frequency & $17 \mathrm{MHz}$ & \\
Cycles & 1.5 & \\
Scanlines & 192 & \\
Emissions per scanline & 2 & \\
Active elements & 64 & SASB \\
\hline Imaging Setup & DRF & $80 / 80$ \\
\hline Focus Tx/Rx (mm) & $85 /$ dynamic \\
F\# Tx/Rx & $5.67 / 0.8$ & $3 / 3$ \\
Apodization Tx/Rx & Boxcar/Gauss & Boxcar/Gauss \\
\hline
\end{tabular}
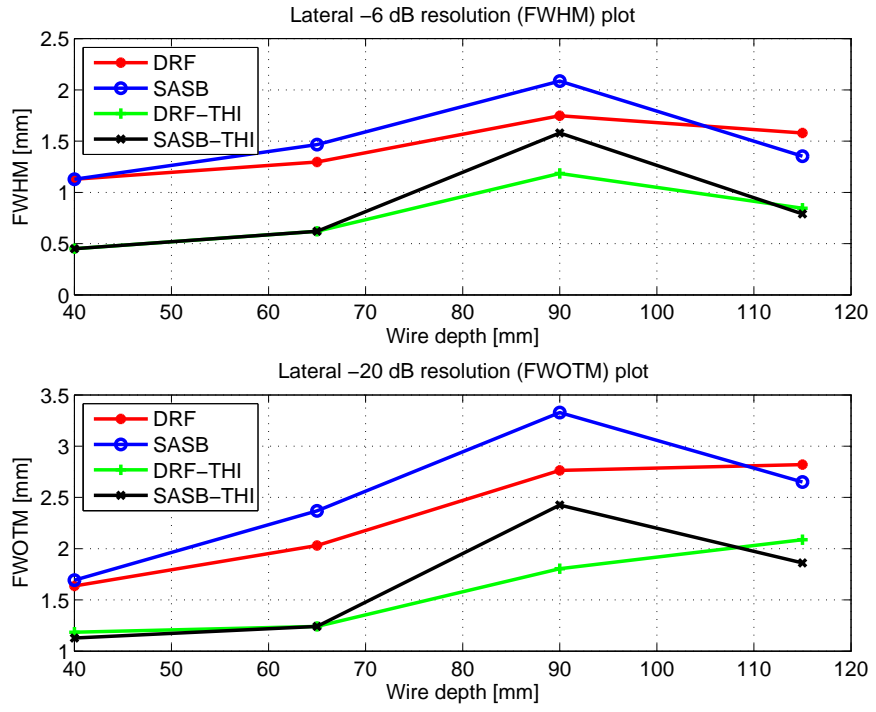

Figure 6. Lateral $-6 \mathrm{~dB}$ resolution (FWHM) and $-20 \mathrm{~dB}$ resolution (FWOTM) of visible wires.
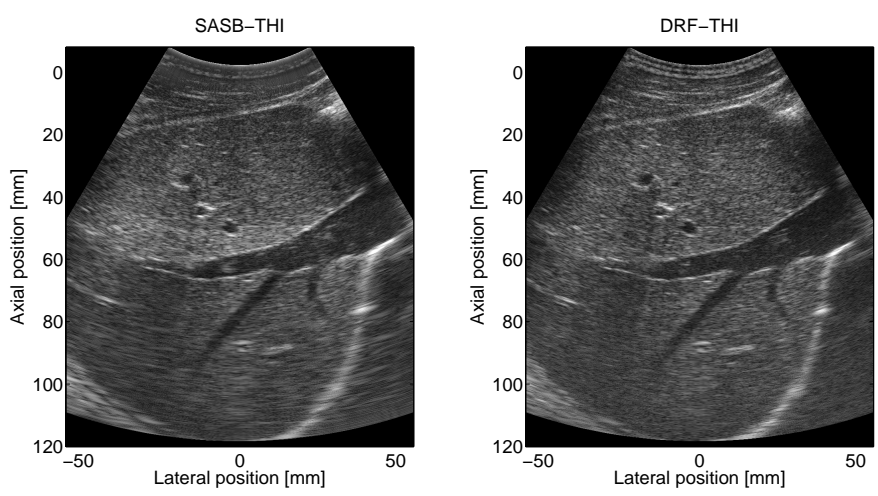

Figure 7. In-vivo image of liver tissues of a 29-year old healthy male volunteer. Left image shows SASB-THI, right image shows DRF-THI.

for the deepest wire at $100 \mathrm{~mm}$. The FWOTM for SASBTHI at $[25,50,75,100] \mathrm{mm}$ is reduced by $[5,0-34,11] \%$ compared to DRF-THI, which shows, that except for the point of the virtual source, the FWOTM is improved by SASB-THI.

2) Comparison of in-vivo Images: Two simultaneously acquired in-vivo images are shown in Fig. 7. The images show a section of liver tissue with blood vessels of a 29year old healthy male volunteer. The left in-vivo image shows the acquired SASB-THI image, while the right image shows DRF-THI. The two images are very similar in appearance. A slightly better penetration depth is acquired using SASB-THI and the edges of the large vessel are more sharp.

\section{DISCUSSION}

The results show as predicted, that non-linear imaging is possible using SASB. The lateral resolution of SASB-THI is improved compared to linear SASB. The lateral resolution of SASB-THI is close to the same as for DRF-THI except at the 
focal point. These results do not show as good improvements to the lateral resolution as the study by Du et. al. [7] showed. This is mainly because, Du et. al. used a linear transducer for SASB-THI, whereas in this study, a convex array transducer is used. The difference between the shape of the transducer has a direct effect on the positions and density of the virtual sources in SASB. Because the image lines spread out with depth using the convex array transducer, fewer image lines are used in the second stage beamformed SASB image at the point of the virtual source. This means that the resolution around the virtual source is not as good with a convex array transducer compared to a linear array transducer.

Furthermore, in the study by Du et al [7], transmit apodization was used. Here, a Hanning was used in transmit which resulted in low levels of side lobes. On the UltraView, no transmit apodization (besides Boxcar) is possible. This causes the level of side-lobes to be greater. But in the case of comparing SASB-THI to DRF-THI on the UltraView, no transmit apodization was used in both cases. This makes the comparison between the two fair, but improvements in the two imaging techniques can be made by applying transmit apodization.

The strengths of this study are, that a commercial ultrasound system is used and that the scan sequence created for this study performs both SASB-THI and DRF-THI simultaneously. The commercial platform allows the testing of SASB-THI in preclinical trials on volunteers and patients. Because SASB-THI and DRF-Image data are acquired simultaneously, comparison of clinical data is much more straight forward. There is only a minimum of time delay between each frame of the two techniques, so the scan geometry is always the same in the two images. Performance evaluations of the two techniques can be made by professional radiologists in double blinded studies as in the study by Hemmsen et. al. [6].

\section{CONCLusion}

SASB-THI has been successfully implemented on a commercial ultrasound system in a scan sequence that also acquires DRF-THI simultaneously. Lateral $-6 \mathrm{~dB}$ resolution for SASBTHI is close to identical to DRF-THI and better than both SASB and DRF. Lateral $-20 \mathrm{~dB}$ resolution is also improved, except at the point of the virtual source. The implementation of SASB-THI on a commercial ultrasound system allows for in-vivo data to be acquired simultaneously with DRF-THI for performance comparisons.

\section{ACKNOWLEDGEMENTS}

This work was supported by grant 024-2008-3 from the Danish Advanced Technology Foundation and BK Medical Aps, Denmark.

\section{REFERENCES}

[1] C. S. Chapman and J. C. Lazenby, "Ultrasound imaging system employing phase inversion subtraction to enhance the image," US Patent, vol. 5632277, 1997.

[2] D. H. Simpson, C. T. Chin, and P. N. Burns, "Pulse inversion Doppler: a new method for detecting nonlinear echoes from microbubble contrast agents," IEEE Trans. Ultrason., Ferroelec., Freq. Contr., vol. 46, no. 2, pp. 372-382, 1999.

[3] J. A. Jensen, "Medical ultrasound imaging, progress in biophysics and molecular biology," Progress in Biophysics and Molecular Biology, $\mathrm{p}$. Submitted, 2006.

[4] M. A. Averkiou, "Tissue harmonic imaging," in Proc. IEEE Ultrason. Symp., vol. 2, 2000, pp. 1563-1572.

[5] J. Kortbek, J. A. Jensen, and K. L. Gammelmark, "Synthetic aperture sequential beamforming," in Proc. IEEE Ultrason. Symp., 2008, pp. 966969.

[6] M. Hemmsen, P. M. Hansen, T. Lange, J. M. Hansen, K. L. Hansen, M. B. Nielsen, and J. A. Jensen, "In vivo evaluation of synthetic aperture sequential beamforming," Ultrasound Med. Biol., vol. 38, no. 4, p. 708Ű716, 2012.

[7] Y. Du, J. Rasmussen, H. Jensen, and J. A. Jensen, "Second harmonic imaging using synthetic aperture sequential beamforming," IEEE Trans. Ultrason., Ferroelec., Freq. Contr., pp. 2261-2264, 2011. 